\title{
Die Indexierung der Welt. Macht und Einfluss einer digitalen Weltmacht
}

\section{Verleihung des rheinland-pfälzischen Datenschutzpreises 2014 im Zeichen von Google und Snowden}

\author{
Zum fünften Mal hat der rheinland-pfälzische Datenschutzbeauftragte am 15. \\ September 2014 seinen Wissenschaftspreis verliehen. Die Veranstaltung stand im \\ Zeichen von Google und dessen Bedeutung bei der aktuellen Medienevolution. \\ Unter dem Titel „Aufklärung im Interesse der Freiheit" wurde anlässlich der \\ Preisverleihung eine Erklärung zu Edward Snowden veröffentlicht.
}

\section{Google - The World Brain}

Zum fünften Mal hat der rheinland-pfälzische Landesbeauftragte für den Datenschutz und die Informationsfreiheit am 15. September 2014 den Datenschutzpreis für herausragende wissenschaftliche Arbeiten im Bereich des Datenschutzes verliehen.

Der Preis ging in diesem Jahr an Dominique Facciorusso für ihre an der Johannes Gutenberg-Universität Mainz erstellte Bachelorarbeit „Google - The World Brain. Was passiert, wenn die ganze Welt zum Index wird", welche die Google-Suchmaschine und die Ambivalenz und individuellen und gesellschaftlichen Konsequenzen der von Google angebotenen Dienste behandelt. Die Arbeit liefert eine profunde Beschreibung von Google und dessen Suchmaschine und zeigt, dass eine Auseinandersetzung mit Google bei aller Würdigung der Vorteile von dessen Diensten eine kritische Distanz zu dem Unternehmen nahelegt.

In ihrem Vortrag „Die Googleisierung der Informationssuche die Macht von Google und die Ohnmacht der Nutzer" stellte Professor Dr. Birgit Stark, Johannes Gutenberg-Universität Mainz, die Ergebnisse einer interdisziplinären Studie über die Google-Suchmaschine vor.

Der Landesbeauftragte für den Datenschutz und die Informationsfreiheit Rheinland-Pfalz, Edgar Wagner, wies darauf hin, dass Google derzeit mit einem Milliardenaufwand an Lösungen zur Künstlichen Intelligenz arbeite, um seine gigantischen Datenmassen besser analysieren zu können. „Während wir noch nach Antworten auf die Fragen unserer digitalen Gegenwart suchen, stellen sich schon die Fragen unserer digitalen Zukunft. Sie sind noch drängender, noch existentieller und noch schicksalhafter als alles, womit wir uns derzeit befassen. Es ist kaum zu verantworten, dass man in diesen Fragen Google grenzenlos vertraut.“

\section{Aufklärung im Interesse der Freiheit}

Unter dem Titel „Aufklärung im Interesse der Freiheit“ veröffentlichte der Landesbeauftragte im Rahmen der Preisverleihung eine Erklärung, mit der die Verdienste Edward Snowdens gewürdigt werden. Ihm sei es zu verdanken, dass in Staat und Gesellschaft eine breite und intensive Diskussion über notwendige Strategien gegen eine alltägliche und globale Überwachung in Gang gekommen sei.

„Dies hat das Datenschutzbewusstsein geschärft und die digitale Welt verändert", so Wagner, der zugleich den Mut und die Zivilcourage Snowdens hervorhob. Mut sei - wie wir seit der Antike wüssten - das Geheimnis der Freiheit. Dies habe Edward Snowden auf eindrucksvolle und lehrreiche Weise bestätigt. Es verdiene festgehalten und gewürdigt zu werden, dass Edward Snowden mit den Mitteln des zivilen Ungehorsams und der Aufklärung dafür streite, dass auch im digitalen Zeitalter Menschenwürde und Freiheitsrechte eine Begrenzung staatlicher und wirtschaftlicher Macht geböten.

Weitere Informationen : http://s.rlp.de/preis2014

\section{Edgar Wagner}

Der Landesbeauftragte für den Datenschutz und die Informationsfreiheit Rheinland-Pfalz 